\title{
INITIAL INTEGRAL PRODUCT AND ASSEMBLY STRUCTURING: A CASE STUDY
}

\author{
J. Küchenhof $\bowtie$ and D. Krause \\ Hamburg University of Technology, Germany \\ $\square$ jan.kuechenhof@tuhh.de
}

\begin{abstract}
Modular product structuring has been proven an effective way to satisfy a high sales variety with low internal product and process complexity but is usually carried out on existing structures. To support the modular new product development, this contribution shows the initial structuring of integrated product and assembly structures based on a variance-oriented product structure. With help of a perspective-based modularization approach, consolidating the product life phases of procurement, assembly and sales, modular product and process structures are developed within an industry case study.
\end{abstract}

Keywords: modularisation, new product development, design for x (DfX), variant management, process structuring

\section{Introduction}

Global competitors, emerging technologies and changing customer requirements lead to innovation pressure in companies. Low cost and high-quality products are required that also satisfy a high variety of customer requirements to reach market diversification. The company Hauni Maschinenbau GmbH, a mechanical engineering company in Hamburg operating in the international tobacco industry, faces those challenges and tries to answer arising market needs by the integration of inventive technologies and innovative product concepts into growing markets. In cooperation with the Institute for Product Development and Mechanical Engineering Design (PKT) of the Hamburg University of Technology, the industry project $\mathrm{NeMo}$ has been initiated to methodically support the new development of modular product families. The Integrated PKT-Approach for Development of Modular Product Families (PKT-approach) is a holistic approach to reduce internal component and process variance while maintaining the external product variety through a modular product strategy (Krause and Gebhardt, 2018). Within the industry project, methodical building blocks of the PKT-approach are adapted and carried out to methodically support the initial development of modular product and process structures. Starting with goal setting and identification of customer requirements, a product structure has been developed. Now, modules have to be formed and production processes need to be set up. This paper shows the initial structuring of integrated product and assembly structures with help of perspective-based modularization, consolidating the product life phases of procurement, assembly and sales within a case study in order to support the new product development (NPD) of modular product families.

The theoretical background regarding established methods for modular product development and methods for structuring the assembly is presented in section 2. The tailored PKT-approach is explained in section 3, 
followed by the compiled modular structures for procurement, assembly and sales. The resulting assembly structure is shown and the methodical procedure as well as its application in the project is discussed. A conclusion and outlook conclude the paper in section 4.

\section{Theoretical background}

A common classification of complexity is the subdivision into external and internal complexity (Lindemann et al., 2009). Lindemann describes external complexity as market-driven and includes standards, laws, competitors, customer-variety, trends, etc.. Internal complexity can be subdivided into the domains: product complexity (with product variants, technologies, etc.) process complexity (division of labor, production, assembly, etc.) and organizational complexity (inhibiting humans, structures, workflows, etc.) (Lindemann et al., 2009). The correlation of external and internal complexity is shown in Figure 1.

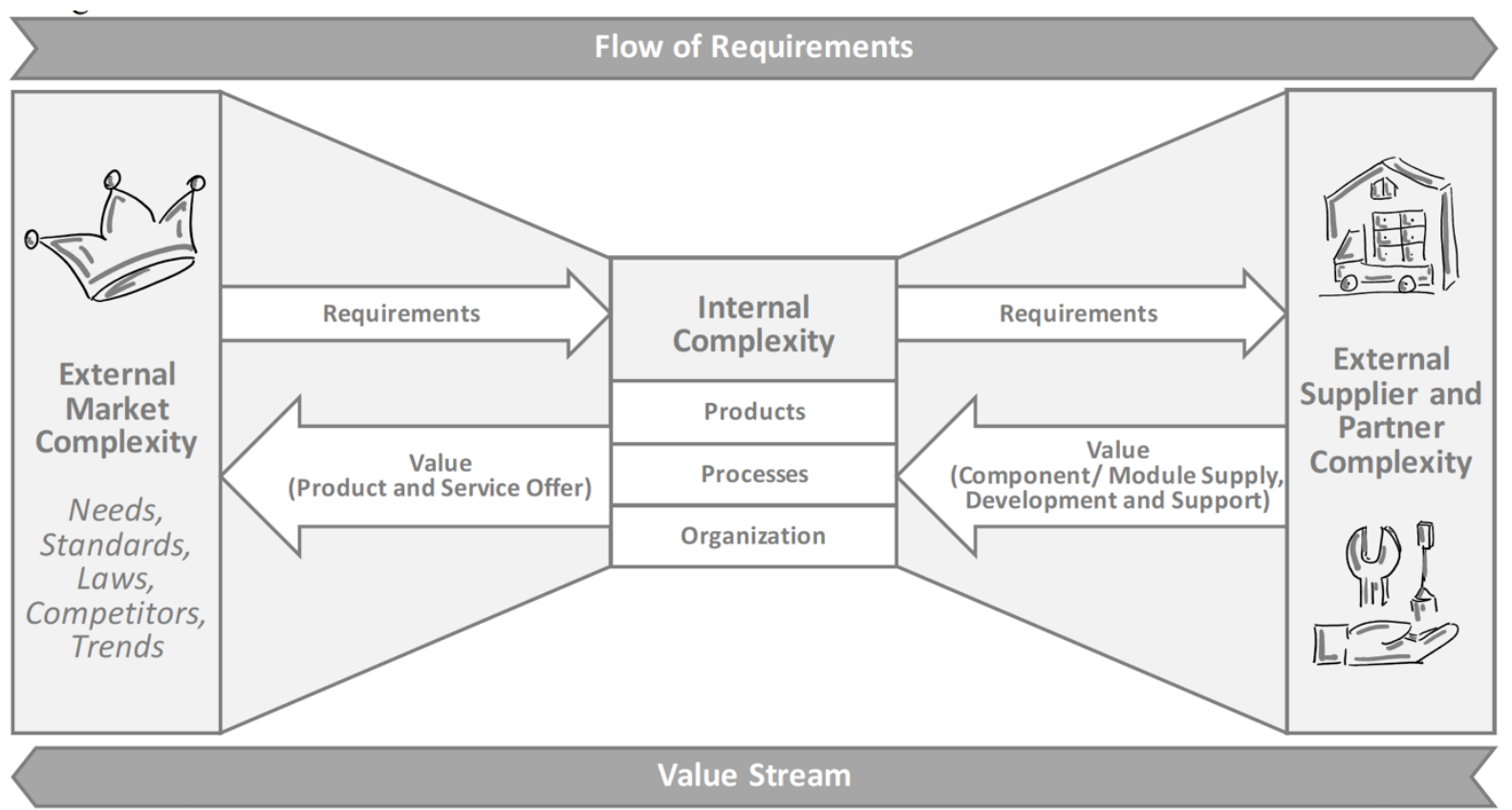

Figure 1. Correlation of external market, supplier and partner complexity and internal component, process and organizational complexity

The company, with the three domains containing internal complexity grows with increasing elements and linkages, is portrayed in the centre. The external market complexity is portrayed on the left, with a high external sales offer as the goal is the proliferation of future product variety. Initial knowledge about products and processes is low within start-up like structures and in order to reduce development lead times, integration of suppliers and development partners has been under special consideration in research (Sanchez, 1996; Chaudhuri and Boer, 2016; Boer and Boer, 2019) and is included to the right from the internal block. The upper arrows indicate the flow of requirements. Users and customers formulate their requirements and needs which have to be taken into account by the producing company and need to be passed on to contracted development partners and suppliers to assure functional reliability and associated product quality. These in turn supply components and can assist the development activities. The arrow down in the picture indicates the value stream from suppliers to the company as the product is produced and transferred to the market. External and internal variety can be seen as a subset of product complexity. Modular product structuring helps to decrease internal variety while maintaining a high sales offer diversity (Krause and Gebhardt, 2018) but is usually carried out on existing product and process structures (Küchenhof and Krause, 2019). The described internal complexity domains are seen as simultaneously growing systems within the new development, where every single one has to be considered. In the following, established methods for product modularization and process structuring are presented and concluded with a brief positioning of modular NPD. 


\subsection{Methods for modular product development}

Modularization methods can be classified into technical-functional and product-strategic approaches. Examples for the former approaches are the Design Structure Matrix where modules are deployed based on the coupling of product components (Pimmler and Eppinger, 1994) or the Module Heuristics after Stone (1997) based on functional structures. Product-strategic approaches take into account requirements from different perspectives during the product life phases. Examples are the Modular Function Deployment (MFD) after Erixon et al. (1996) and the Life Phase Modularization (LPM) after Krause and Gebhardt, (2018). The general procedure of modularization can be comprehended as the (1) decomposition of the existing product structure, the (2) analysis of components, the (3) modularization in the selected observation level and the (4) transfer in a new modularized product structure (Krause and Gebhardt, 2018). The PKT-approach incorporates technical-functional as well as product-strategic modularization in order to technically develop product families but also takes into account different perspectives during product life phases to optimize the modular product structure for each life phase (Krause and Gebhardt, 2018). With the goal of taking different perspectives and requirements into account, LPM tries to achieve a harmonized modular product structure. The Module Process Chart (MPC) is the main visual product development tool to integrate the different views and to harmonize the resulting modular structure. In the MPC, product components are listed for each considered product life phase. The component flow into and separation from modules is made visible throughout the life phases. LPM proposes that modules should grow passing through the life-phases as value increases until the product is treated as one final sales module. Net plans are used to support module formation for each life phase. Similar to the MFD method, fitting modular drivers and driver characteristics of the concerned product-life phase need to be identified and are then mapped to the product components. Seeing the different influences on the components, those with fitting module driver profiles are clustered into modules and transferred to the MPC. Further discussions of possible changes to the product or process design with the relevant stakeholders attempt to harmonize the modular structure throughout the considered product life phases (Krause and Gebhardt, 2018).

\subsection{Assembly process structuring}

With focus on an integral product and assembly structure, Halfmann focusses the product assembly within manufacturing (Halfmann and Krause, 2010). This phase needs special consideration as high costs incur in production, but costs are accounted already during development (Gairola, 1981). Halfmann identifies four major objectives to product modularization with respect to production as reduction of lead times, economies of scale, reduction of the amount of defective goods and customization at a late stage of production (Halfmann et al., 2011). The integrative product and assembly structure (iPAS) method for assembly modularization tries to approach those goals, uniting the integrative view of the product and its assembly process based on the generic product process structure by Jiao et al. (2007). Jiao describes the development of a generic platform encompassing products and processes to encounter product variety with processual standard routings (Jiao et al., 2007). Structuring measures for Design for Assembly proposed in design guidelines can be divided into the categories reduce, standardize, simplify and structuring (Pahl and Beitz, 2007). Halfmann developed heuristics for modular product structuring to integrate these proposed measures (Halfmann et al., 2011). He addresses the measure reduce by avoiding idle times between process steps in order to reduce assembly lead-time and optimizing the capacity of the related assembly system. Simplifying is achieved by grouping components with similar assembly operations into the same module. As for structuring, processes are to be parallelized. This can be reached by combining product components into modules.

Customization at a late stage of production is also correlated to the term postponement. Postponement is a strategy whereby the final configuration of a product is delayed as much as possible (Lee and Billington, 1994). Postponement can help structuring simple tasks such as packaging, labeling or more complex tasks such as localization, assembly and test and final product integration (Lee and Billington, 1994). There is the general differentiation into time postponement and form postponement. Time postponement describes the delay of tasks of product differentiating and other activities (labeling, assembly, etc.) whereas form postponement tries to standardize the upstream stages as much as possible and defer the point of product differentiation. Form postponement often requires redesigning the product with increased modular structure and component commonality (Lee and Billington, 1994). Associated benefits to implementing 
postponement is reduced inventory costs, ease of product upgrades and shorting NPD lead times, which can have significant market value (Lee and Billington, 1994). Together with process-commonality, Kipp proposes postponement as a process strategy with regard to product variety.

\subsection{Modular new product development}

NPD can be understood as the process of developing, producing and developing inventive products to the market (Chaudhuri and Boer, 2016). The main difference between modular NPD and traditional NPD methods is that the product architecture, as the linkage from functional elements to product components (Ulrich, 1995), is input to the development activities and not the result of them (Sanchez, 1996). Support for the initial development of modular product structures is given in Küchenhof and Krause (2019) with a future-oriented approach of Design for Variety (DfV). Consequently, the derivation of processes based on the planned product structure is highlighted in this contribution. As well as in product structuring, case studies of initial process structuring in entrepreneurial environments are hard to find. This case study provides insights on the early phase development activities, handling future uncertainty projected on certain, flexible modules and corresponding process steps. A generic product structure, derived within a product architecture (Küchenhof and Krause, 2019) builds the basis for following LPM, including assembly structures, which is shown in the following.

\section{Case study}

The industrial project NeMo comprises the methodical support in product, process and organizational structuring for initial development activities of new generation products. As this project contains both, an industry sided problem base and a theory base by the involvement of the university, an action research approach according to Jørgensen (1992) is chosen, as the researchers' interest is not only to observe but also influence and control the case. Therefore, models of the PKT-approach are altered and applied within the new development project. The product and process domain are attended by tailoring the PKT-approach towards initial structuring for future product variety. Instead of recording external and internal variety, the related methods steps and models are changed towards planning activities. The altered development approach, subdivided into four phases, is shown in Figure 2.

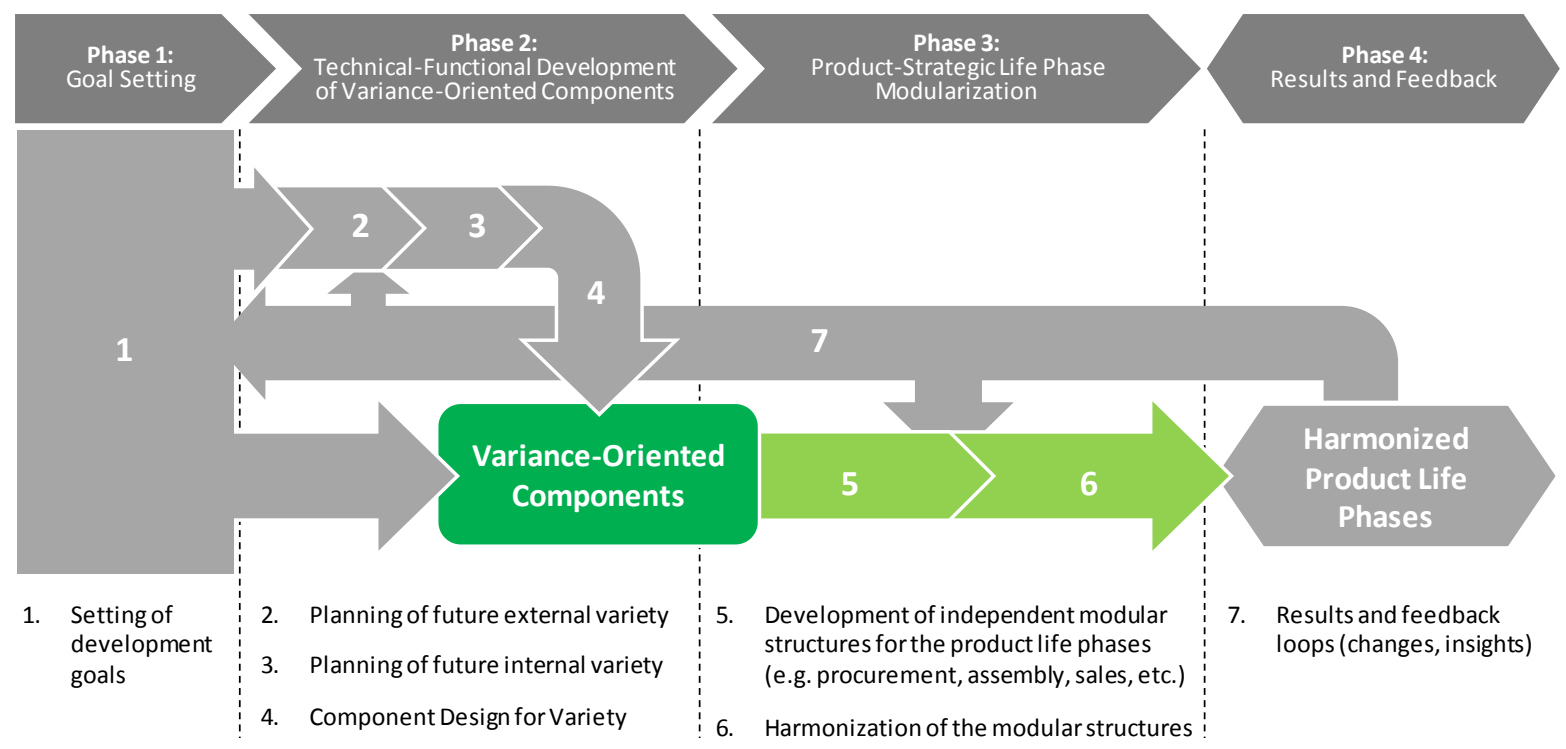

Figure 2. Integrated PKT-approach tailored to new product development within the industry project NeMo

Phase 1 is attributed to goal setting. Within the development project, the superior development goals are formulated as high flexibility, short time to market, short product development lead times, low cost components and high quality. In phase 2 variance-optimized components are to be developed based on technical-functional measures. Therefore, the future external and internal variety need to be planned (step 2 and 3) and in step 4, variance-oriented components are developed. This is the starting basis for subsequent 
product life phases and is highlighted in green. Perspective-based LPM is carried out in phase 3. In the following, the focus is on assembly within the production phase as part of LPM. The results of the development activities are gathered in phase 4. This phase is supplemented by feedback loops (step 7) to earlier development phases in order to counteract the high dynamics in the early phase and retract insights during development activities to previous development stages. Based on variance-oriented components, step 5 and 6 within phase 3 are focal point of the following consideration and are marked in light green. Although all product life phases need to be integrated for a fully optimized product structure, the preceding and subsequent life phases of assembly, procurement and sales, are emphasized for initial structuring. The results are shown in the earlier introduced MPC and net plans as well as the iPAS, which is complemented by test elements. The generic product structure consists of main product system attributes such as system boundaries, electrical, mechanical and hydraulic interfaces. Due to non-disclosure agreements and proprietary contents, not all information of the real development case can be displayed or must be abstracted.

\subsection{Initial harmonization of the modular product structure throughout the product life phases}

The results from step 5, development of independent modular structures for the product life phases and step 6, harmonization of the modular structures, are displayed in Figure 3. The MPC and appurtenant net plans for the life phases procurement, assembly and sales are shown. The identified company specific module drivers and its characteristics are elucidated and suitable module formations are motivated. Afterwards, the modular structure is shown in the harmonized MPC. The product components are differentiated into standard (white), variant (grey) and specific (blue). The evaluation of the probability of future component variations result from phase 2 as part of the future-oriented DfV.
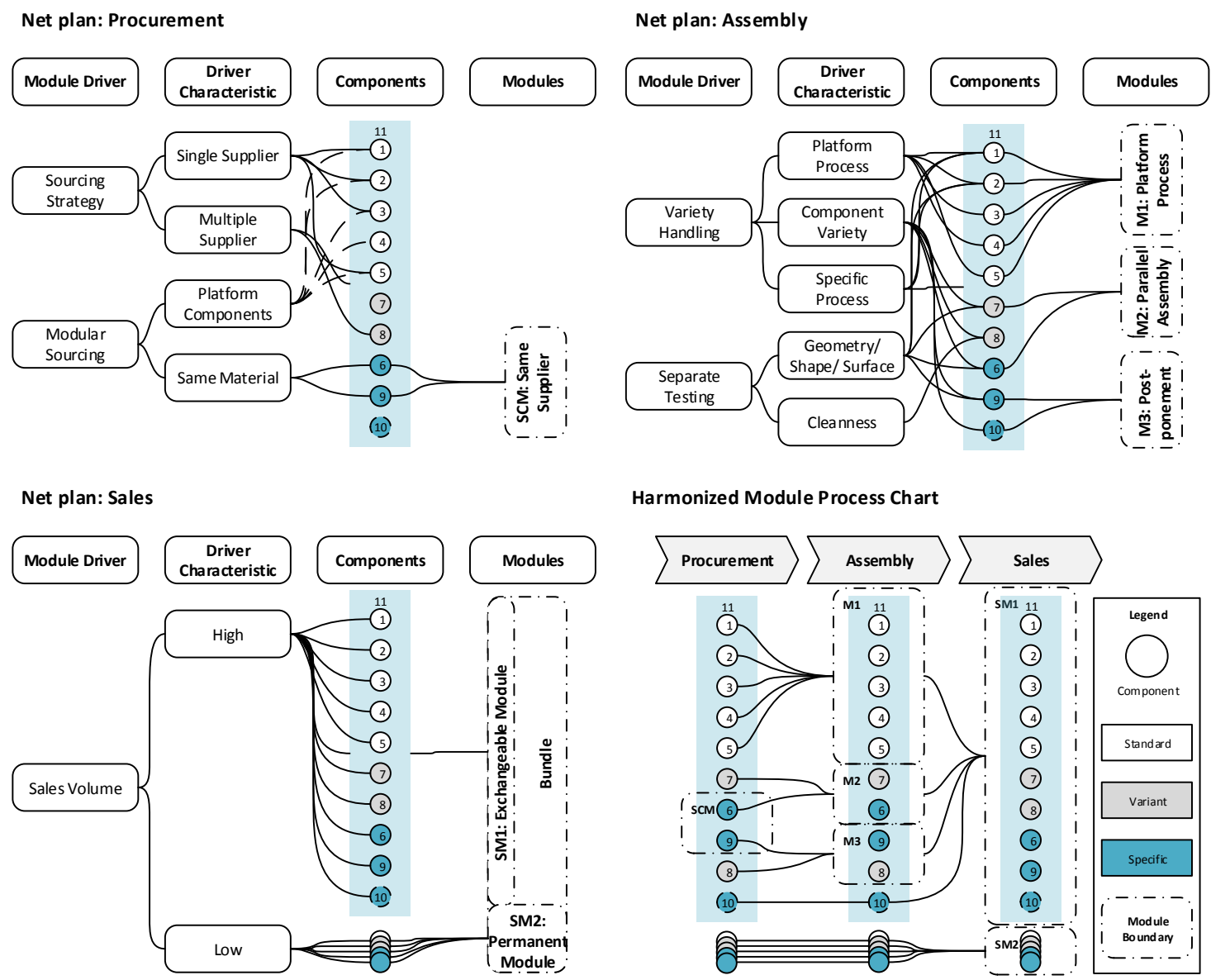

Figure 3. Netplans for the considered product life-phases procurement (top left), assembly (top right) and sales (bottom left) and harmonized module structure in MPC (bottom right) 
Net plan: Procurement (Figure 3, top left): The first identified module driver of the procurement phase is sourcing strategy with its characteristics single supplier and multiple supplier. The procurement has to steer the input for internal manufacturing and coordinate suppliers with components being purchased. Legal responsibilities and contracting in terms of quality, reliability, etc. are important as well as the strategical orientation regarding regional coordination and securing a consistent and reliable supply-chain through e.g. a multiple supplier strategy for certain components. An important differentiation has to be made for suppliers during prototyping (which are needed to support the component design and prepare for manufacturing) and serial production. Low volume orders are usually more expensive, lacking economies of scale, but know-how for handling certain materials or processes is needed to be integrated. Cost estimations comparing prototype designs and serial components can differ greatly and need to be treated sensitively. Ideally, suppliers that are incorporated early on can prove to be valuable development partners that are continuously involved and developed to later production partners. The other identified module driver is modular sourcing with the characteristics platform components and same material. Components linked to those module drivers can eventually be bought as procurement modules. As the procurement phase is still very fuzzy, it is challenging to find suppliers for prototyping with the capability to produce designs in appropriate time and quality. Thus, only the two specific components 6 and 9 are considered for initial modularization as the SCM procurement module with one supplier for both components.

Net plan: Assembly (Figure 3, top right): The assembly unites the production of own components, the integration of supplier components and modules as well as labeling and packaging to prepare for distribution. During production, the assurance of quality measures is of high importance and can be ensured by appropriate tests. The first identified module driver variety handling is assigned with the driver characteristics platform process, component variety and specific process. As described earlier, it can be beneficial in terms of standardization to mirror product platform components with platform processes. Separate testing is also accommodated as a module driver with the geometrical characteristics geometry/ shape/ surface and the chemical testing characteristic cleanness. Three assembly modules are formed resulting in assembly module M1 which inhibits the product platform components, module M2 that is separated for parallelizing process steps and the process module M3 for the highly customer specific packaging process. Further details on the modular assembly structure is given within the following process structuring using the iPAS method.

Net plan: Sales (Figure 3, bottom left): The most relevant module driver for sales in this case is sales volume with the driver characteristics high and low. Due to the functional use of the product during usage, a physical cut can be found in the product structure. All components considered for assembly are influenced by a high sales volume with approximately 100 times more units produced and sold than the other components, which is indicated in the sales net plan. The two resulting modules are the exchangeable module SM1 and the permanent module SM2. There is also the option of selling the permanent and exchangeable modules in a bundle with more than one exchangeable unit. The exchangeable unit SM1 defines the scope of the presented LPM and process structure, as more revenue is involved in relation to this module and it is therefore more critical for the business case.

Harmonized Module Process Chart (Figure 3, bottom right): The resulting modular structures from the net plans for the considered life phases are transferred to the MPC. The flow of components to and from modules over the product life phases are visible and inconsistencies in between can be recognized. It is noticeable, that the SCM module, consisting of the highly specific components 6 and 9, need to be separated during assembly. This may seem displeasing but makes sense considering preceding product life phases as this module is also correlated to haptic and visual requirements and is thus treated as one design unit in product development already (which is not shown here). Both components also need to run through the same testing before assembly as can be deduced in the net plan for assembly. Module assembly takes place successively and the packaged product is then to be distributed and transferred to the sales phase as one module. The separate assessment for the permanent module in sales has also been carried out but is not shown here.

\subsection{Integral product and process structuring}

Next, the assembly processes are set up and structured following the iPAS method on the basis of the different modular product structures developed for the life phases. The general idea is portrayed in Figure 4, which is separated into the three parts Pre-Assembly, Assembly and Post-Assembly. While assembly is emphasized, 
pre-assembly is included in the assembly activities and taken into account in the product life phase procurement. Post-Assembly as the output of the assembly sequences is considered by including the subsequent product life phases such as sales. The division into standard, variant and specific parts is also carried out for processes. While in standard processes no process variation is necessary, with variant processes e.g. parameter changes or tool changes are taken into account. Specific processes are regarded as customerspecific and require a great deal of change effort for specific component variants. The processes are connected by white circles. The following presentation of the developed process chains is based on this diagram.

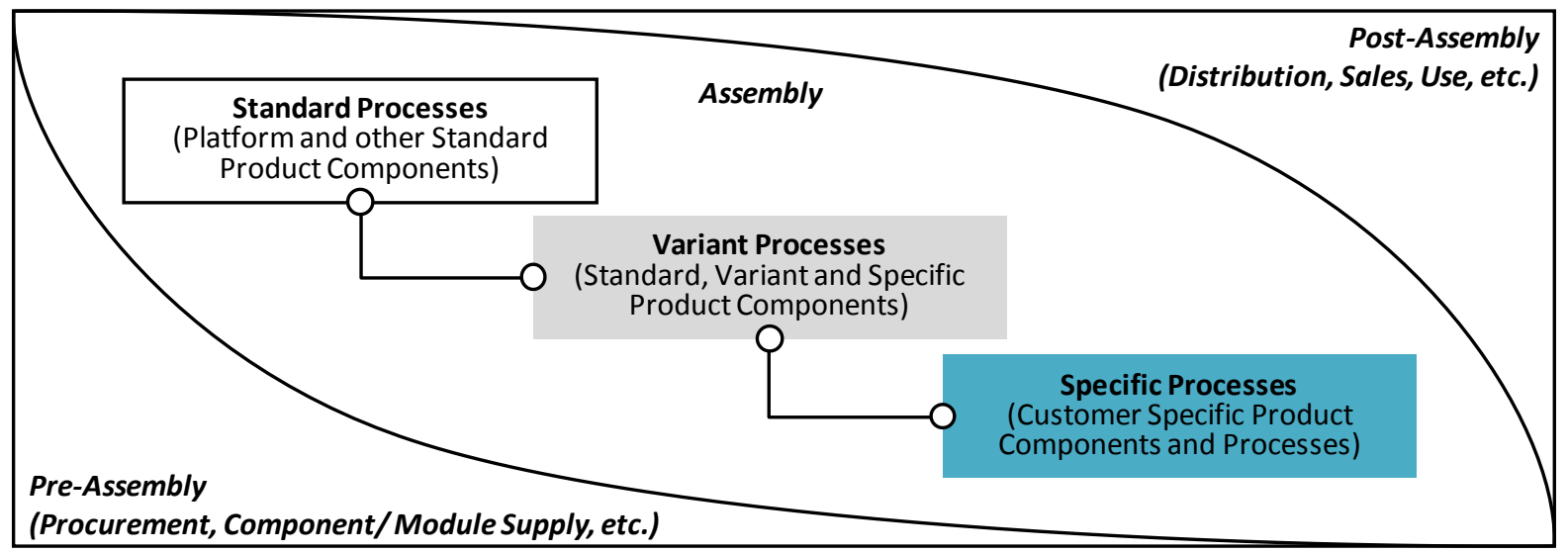

Figure 4. Variety allocation in assembly processes within the scope of Pre- and Post-Assembly

The iPAS elements used and added are briefly explained according to the segmentation in Figure 5. The product elements used are components and their linkages which can be mechanical (orange), electrical (green) or material (blue) connections. The differentiation into standard (white), variant (grey) and specific (blue) components is made, which is also used for process characterization. Further, the product elements are integrated into the process elements. There are base process cards and general process step cards. Both are filled with information, originating from the product and process side. Operating equipment, process activities and materials are inserted. Supplementary components are inserted and the growing product structure during the process is visualized in the corresponding field. To integrate the testing view, testing elements are introduced, answering the questions where components are tested, who is responsible, which test is conducted and how many samples are inspected. Tests are recorded in the show $\mathrm{n}$ pentagon. In the roof, the test type, which can be optical, electrical, mechanical or market tests and the test responsibility are mapped. Tests can take place before assembly (light purple), during assembly activities (light green) or after assembly (light orange). The sampling ranges from random sampling (red) and systematic sampling (orange) to total control (100\%) tests (green) where each object is individually tested.

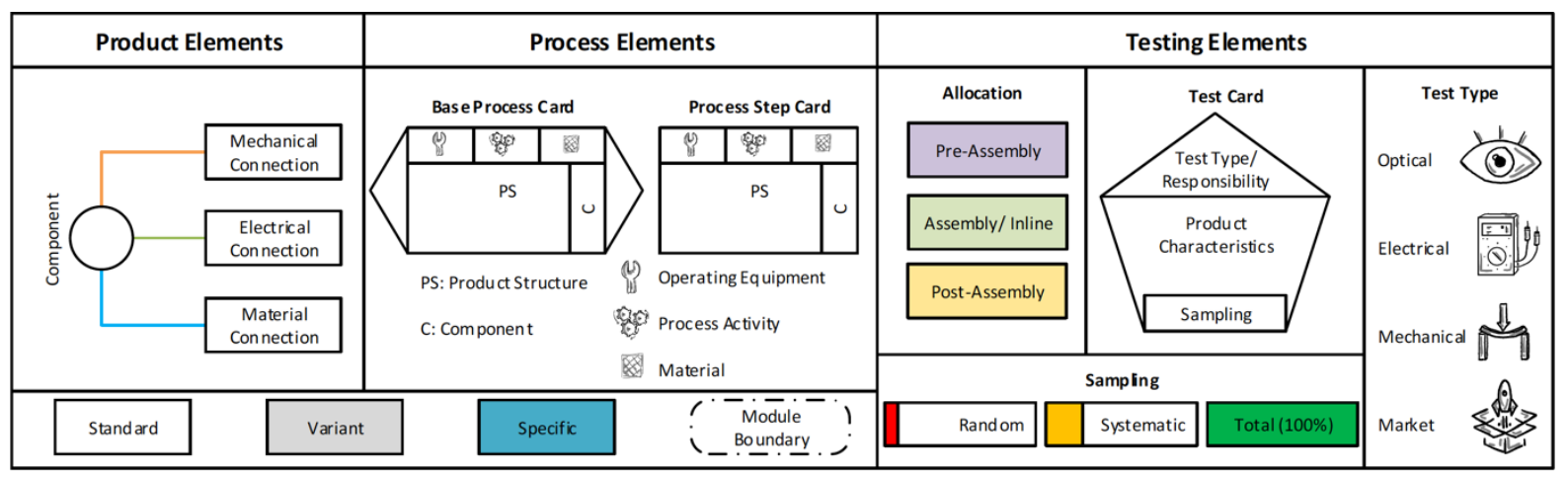

Figure 5. Product, process and testing elements used for process structuring 
The three resulting process chains for the sales module SM1 are shown and explained in the following.

Standard Processes: Figure 6 shows the identified platform process for the given modular product platform. The process chain is separated into two serial sequences. The basis component 1 is connected to component 2 in an excluded, separate process, as this process step is identified complicated and critical for internal quality measures. Therefore, a $100 \%$ test is implemented before more platform components are added in the subsequent platform process. Due to a possible and likely spatial separation of the complicated process and the platform process, the merged components need to be tested after transportation and the test is executed as a first step of the second process chain and not at the end of first one. The green color in the test box indicates an optical and mechanical inline test for cracks and fractures for all components (100\%). The consecutive process steps finalize the platform module M1, merging the standard components by means of standard process steps, standard tools and standard materials. The finalized module is then handed over to the next process chain, indicated by the white circle.

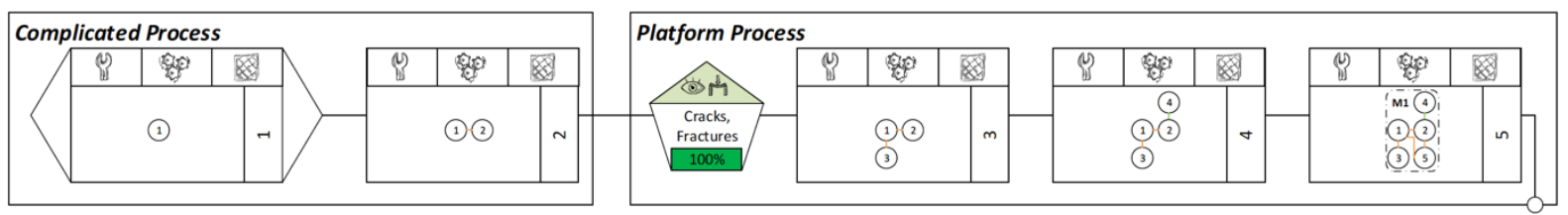

Figure 6. Excluding complicated activities and platform process

Variant Processes: The variant process sequence is portrayed in Figure 7. The procurement module (SCM), consisting of the highly customer specific components 6 and 9, is purchased from the same supplier (see Figure 3, top left). Both components are tested optically for shape and surface characteristics (such as dimensions, tolerances, texture). A defined sample size is extracted from each produced batch depending on statistical significance (e.g. 5 per batch). The supplier is responsible for component and manufacturing process quality as indicated by the purple roof. The components are entered separately, enabling parallelizing subsequent process steps. M2 is assembled first by adding component 7 . The platform module M1 is then connected to M2 and component 8 is added. Meanwhile, M3 is assembled in parallel by merging component 10 . M1 and M2 are now joined with M3. It can be seen in the product structure that the electrical connection is established by now. Accordingly, a circuit test can and will be performed for all assembled modules (100\%) within the machine (green roof). It is visible that the variant process inhibits standard, variant and specific components and standard and variant process steps as indicated in the boxes within the iPAS elements. This process sequence is labeled as variance-robust because it is designed to handle the estimated component diversity.

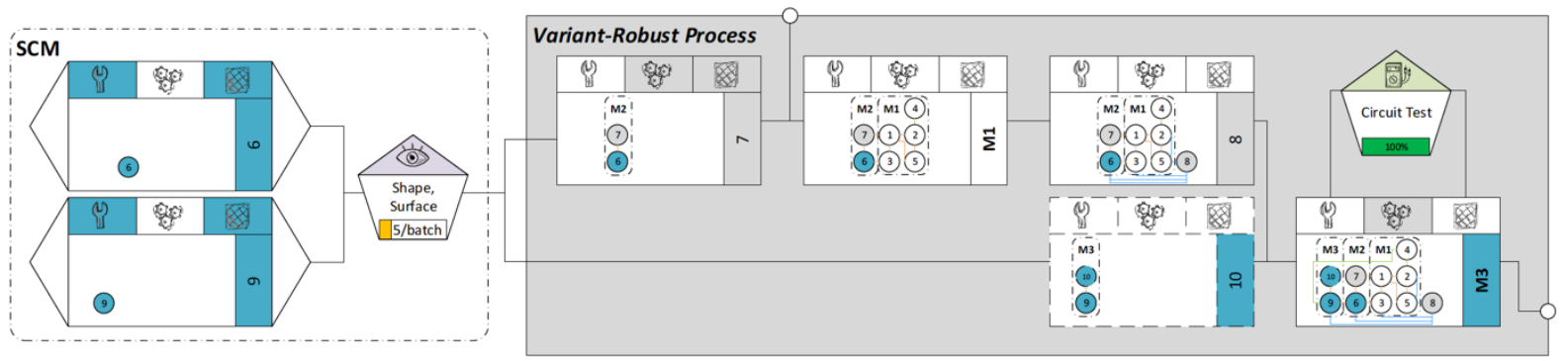

Figure 7. Module procurement, testing and variant-robust process

Component 10 is used for labeling which is not necessary for each customer but if, it is accompanied by very specific requirements and therefore rated as a highly customer specific, optional component.

Specific Processes: The last presented process sequence can be seen in Figure 8 and is marked as highly specific. It incorporates the packaging of the assembled components and modules. During the development, it could be verified (as proposed by Lee and Billington, 1996), that packaging can be very different in terms of size and type of packaging for each customer. As a result, this process step is separated. A high rearrangement of the machine or new development would be necessary. After packaging, the exchangeable sales module SM1 can be used for product testing and pilot studies on the market, which 
is indicated by the orange roof and the product symbol. The tested sample size is allocated at the bottom with $\mathrm{n}=100$ for product tests on the market.

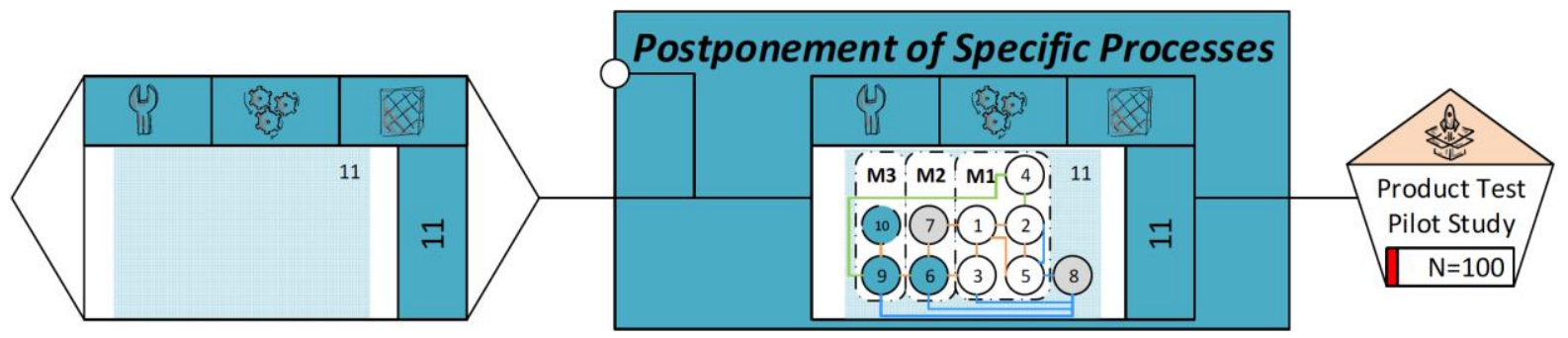

Figure 8. Specific packaging process

In summary, it can be said that some of the structuring measures described in the literature have been successfully implemented. Parallelization is reached by the subdivision of the whole process in separate machines and the concurrent assembly of modules within machines. Meaning, that final assembly can take place at a later point of time or assembled modules can be put in stock. Postponement is achieved by deferring the point of product differentiation to later process steps, reducing the assembly effort and increasing process flexibility. The modular product structure enables the separation of certain process steps which are self-contained and can be distributed temporally and spatially. Efficient equipment utilization is reached by excluding costly process steps (e. g. to suppliers) and parallelization as assembly time can be reduced. Integral design helps to facilitate some process steps or to omit them completely. The shown process structure is result from many iterations and has been worked out in cooperation with project members from marketing, product development and manufacturing.

\subsection{Discussion}

The shown process is structured with aim to satisfy a high external product variety with low internal component variety and process complexity based on a modular product structure. The project partner and students were able to apply the altered method effectively. It is visible that the point of product differentiation could be deferred to later process steps already during the initial planning, as the standardized platform process builds the basis for assembly and variant components are introduced in later process steps. However, product and process are closely linked. Unplanned component variation, especially in platform components will increase overall complexity, as it is basis for many subsequent product component relationships and processual activities. Ideally, variant or specific product components can be handled with predefined processes through over dimension of tools or machine layout or in-process adaptions to variant parameters (e.g. length variation), creating a component variance-robust process. In order to assess how far-sighted and efficient the methodical framework for product and process has been developed, the costs of process change due to additional component variety could be recorded. A great advantage that can be deduced from the methodical procedure is, that product and process design can take place before physical implementation is realized. Restructuring existing processes is often associated with high expenditure and it can be said from own experience, that many suitable product concepts fail due to established structures that are costly to change.

\section{Conclusion and outlook}

In this contribution, the initial structuring of integrated product and assembly structures based on a variance-oriented product structure is shown within an industrial case study. Perspective-based LPM is carried out focussing the assembly phase within production. Under consideration of the preceding and subsequent life-phases procurement and sales, independent modular structures are created and harmonized with help of net plans and the MPC. Subsequently, assembly structures are derived using the iPAS method, which is supplemented by test elements for quality assurance. Modular structuring enables measures such as parallelization and postponement, lowering assembly efforts and leveraging flexibility in production among others. Since product design accounts for a high proportion of product life cycle costs, especially during production (Gairola, 1981), it is regarded necessary to consider requirements 
from different perspectives as early as possible and to incorporate them into product development and design. In order to shorten development lead times and increase internal know-how, supplier and development partner integration has been focal point of some research in the field of NPD (Chaudhuri and Boer, 2016; Boer and Boer, 2019). Analyzing the net plan from the procurement phase (Figure 3, top left), high uncertainty regarding suppliers can be identified. It can be reported from the industry project, that finding the right collaboration partners is challenging. Some do not bring in the right expertise for component development and delivery times can be quite long, while fulfilment of the stated requirements is not always given. Modular structuring creates information structures that facilitate supplier and partner integration (Sanchez, 1996) and enable black-box engineering (Krause and Gebhardt, 2018). Integrating those views via the concept of module drivers is seen as a possibility, consolidating the many distributed responsibilities which require a high level of communication effort.

\section{Acknowledgements}

The authors thank Hauni Maschinenbau GmbH for the interesting collaboration within the industrial project and the knowledge gained through the joint work and the high degree of mutual trust.

\section{References}

Boer, H. and Boer, H. (2019), "Design-for-variety and operational performance: The mediating role of internal, supplier and customer integration”, Journal of Manufacturing Technology Management, Vol. 30 No. 2, pp. 438461. https://doi.org/10.1108/JMTM-03-2018-0065

Chaudhuri, A. and Boer, H. (2016), "The impact of product-process complexity and new product development order winners on new product development performance: The mediating role of collaborative competence", Journal of Engineering and Technology Management, Vol. 42, pp. 65-80. https://doi.org/ 10.1016/j.jengtecman.2016.10.002

Erixon, G., von Yxkull, A. and Arnström, A. (1996), "Modularity - the Basis for Product and Factory Reengineering", Annals of the CIRP Vol. 45 No. 1, pp. 1-6. https://doi.org/10.1016/S0007-8506(07)63005-4

Gairola, A. (1981), Montagegerechtes Konstruieren: Ein Beitrag zur Konstruktionsmethodik, [PhD Thesis], Technical University of Darmstadt.

Halfmann, N. and Krause, D. (2010), "Towards Innovative Assembly Concepts: Integral Product- and Assembly Structure", Proceedings of the DESIGN 2010 / 11th International Design Conference, Dubrovnik, Croatia, May 17-20, 2010.

Halfmann, N., Elstner, S. and Krause, D. (2011), "Product and Process Evaluation in the Context of Modularization for Assembly", Proceedings of the ICED 11 / 18th International Conference on Engineering Design, Lyngby/ Copenhagen, Denmark, August 15-19, 2011.

Jiao, J., Zhang, L. and Pokharel, S. (2007), "Process Platform Planning for Variety Coordination From Design to Production in Mass Customization Manufacturing", IEEE Transactions on Engineering Management, Vol. 54 No. 1, pp. 112-129. https://doi.org/10.1109/TEM.2006.889071

Jørgensen, K. (1992), “Videnskabelige arbejdsparadigmer”, Institute for Production, Aalborg Universitet, Denmark.

Krause, D. and Gebhardt, N. (2018), Methodische Entwicklung modularer Produktfamilien - Hohe Produktvielfalt beherrschbar entwickeln, Springer, Berlin. https://doi.org/10.1007/978-3-662-53040-5

Küchenhof, J. and Krause, D. (2019), "Entwicklung eines Produktarchitekturmodells zur Ableitung modularer Produktfamilien", Proceedings of the 30th Symposium Design for X / DFX 2019, Jesteburg, Deutschland, September 18-19, 2019. https://doi.org/10.35199/dfx2019.3

Lindemann, U., Maurer, M. and Braun, T. (2009), Structural Complexity Management: An approach for the field of product design, Springer, Berlin. https://doi.org/10.1007/978-3-540-87889-6

Lee, H.L. and Billington, C. (1994), "Designing Products and Processes for Postponement", In: Dasu, S., Eastman, C. (Ed.), Management of Design, Springer, Dordrecht, pp. 105-122. https://doi.org/10.1007/978-94-011-1390-8_6

Pahl, G. and Beitz, W. (2007), Engineering Design: A Systematic Approach, Springer, Berlin. https://doi.org/10. 1007/978-1-84628-319-2

Pimmler, T.U. and Eppinger, S.D. (1994), "Integration Analysis of Product Decompositions", ASME International Engineering Technical Conferences, Minneapolis, USA, 1994.

Sanchez, R. (1996), "Strategic Product Creation: Managing New Interactions of Technology, Markets and Organizations", European Management Journal, Vol. 14 No. 2, pp. 121-138. https://doi.org/10.1016/02632373(95)00056-9

Stone, R.B. (1997), Towards a Theory of Modular Design, [PhD Thesis], The University of Texas at Austin.

Ulrich, K. (1995), "The role of product architecture in the manufacturing firm", Research Policy, Vol. 24, pp. 419440. https://doi.org/10.1016/0048-7333(94)00775-3 